KYUNGPOOK Math. J. 51(2011), 251-260

http://dx.doi.org/10.5666/KMJ.2011.51.3.251

\title{
Certain Class of Multidimensional Convolution Integral Equa- tions Involving a Generalized Polynomial Set
}

\author{
Jamal Mohammed Shenan and Tariq Omar Salim* \\ Department of Mathematics, Al-Azhar University-Gaza, P. O. Box 127\%, Gaza, \\ Palestine \\ e-mail : shenanjm@yahoo.com and trsalim@yahoo.com
}

ABSTRACT. The aim of this paper is to obtain a solution of a certain multidimensional convolution integral equation of Fredholm type whose kernel involves a generalized polynomial set. A number of results follow as special cases from the main theorem by specifying the parameters of the generalized polynomial set.

\section{Introduction}

Agrawal and Chaubey [1, p.1155]; see also Srivastava and Manocha [11, p.447] studied the following general class of polynomials

$$
\begin{aligned}
& R_{n}^{\alpha, \beta}[x ; A, B, C, D ; p, r ; q, c ; \omega(x)] \\
= & \frac{\left(A x^{p}+B\right)^{-\alpha}\left(C x^{r}+D\right)^{-\beta}}{K_{n} \omega(x)} \times T_{k, l}^{n}\left\{\left(A x^{p}+B\right)^{\alpha+q n}\left(C x^{r}+D\right)^{\beta+c n} \omega(x)\right\}
\end{aligned}
$$

with differential operator $T_{k, l}^{n}$ being defined as

$$
T_{k, l} \equiv x^{l}\left(k+x D_{x}\right),
$$

where $D_{x} \equiv d / d x$

In (1.1), $\left\{K_{n}\right\}_{n=0}^{\infty}$ is sequence of constants, and $\omega(x)$ is independent of $n$ and differentiable an arbitrary number of times.

Assuming that $K_{n}=1$ and $\omega(x)=1$. Moreover, setting $p=D=1, C=-\tau$ in (1.1) and replacing $\beta$ by $\beta / \tau$ therein, we arrive at the following generalized polynomial set

$$
\begin{aligned}
& S_{n}^{\alpha, \beta, \tau}[x ; r, c, q, A, B, k, l] \\
= & (A x+B)^{-\alpha}\left(1-\tau x^{r}\right)^{-\beta / \tau} T_{k, l}^{n}\left\{(A x+B)^{\alpha+q n}\left(1-\tau x^{r}\right)^{\beta / \tau+c n}\right\} .
\end{aligned}
$$

* Corresponding Author.

Received February 12, 2007; accepted March 18, 2008.

2010 Mathematics Subject Classification: Mathematics Subject Classification: 45B05, $33 \mathrm{C} 60$.

Key words and phrases: Convolution integral equation, generalized polynomial set, Fox's $\mathrm{H}$-function. 
This polynomial set has been studied by Raizada [8].

It may be remarked here that the generalized polynomial set is very general in nature and it unifies and extends a number of classical polynomials introduced and studied by various researches such as Chatterjea [2], Gould and Hopper [4], Krall and Frink [5], Srivastava and Singhal [14], etc. Some of special cases of (1.3) are given by Riazada in tabular form [8; p.65], see also Saigo, Goyal and Saxena [9].

Motivating essentially by the method used earlier by Srivastava and Panda $[12,13]$, the main object of this paper is to present an exact solution of the following multidimensional convolution integral equations of Fredholm type

$$
\int_{0}^{\infty} \ldots \int_{0}^{\infty} y_{1}^{-1} \ldots y_{m}^{-1} u\left(\frac{x_{1}}{y_{1}}, \ldots, \frac{x_{m}}{y_{m}}\right) f\left(y_{1}, \ldots, y_{m}\right) d y_{1} \ldots d y_{m}=g\left(x_{1}, \ldots, x_{m}\right),
$$

where $x_{i}>0, \forall i=1, \ldots, m, g$ is a prescribed function, $f$ is an unknown function to be determined, and the kernel $u$ is given by

$$
\begin{aligned}
& u\left(x_{1}, \ldots, x_{m}\right) \\
= & \prod_{i=1}^{m}\left(A_{i} x_{i}+B_{i}\right)^{\alpha_{i}}\left(1-\tau_{i} x_{i}^{r_{i}}\right)^{\beta_{i} / \tau_{i}} S_{n_{i}}^{\alpha_{i}, \beta_{i}, \tau_{i}}\left[x_{i} ; r_{i}, c_{i}, q_{i}, A_{i}, B_{i}, k_{i}, l_{i}\right] \\
= & \prod_{i=1}^{m}\left[x_{i}^{l_{i}}\left(k_{i}+x_{i} D_{x_{i}}\right)\right]^{n_{i}}\left\{\left(A_{i} x_{i}+B_{i}\right)^{\alpha_{i}+q_{i} n_{i}}\left(1-\tau_{i} x_{i}^{r_{i}}\right)^{\beta_{i} / \tau_{i}+c_{i} n_{i}}\right\} .
\end{aligned}
$$

Throughout this paper, we assume $l_{i}, n_{i}, \alpha_{i}+q_{i} n_{i},(i=1,2, \ldots, m)$ to be nonnegative integers.

Our method of solution of the integral (1.4) with kernel given by (1.5) would depend on the theory of multidimensional Mellin transform defined by $[12$, partI. p.125, eq.(3.5.1)]

$$
M\left\{f\left(x_{1}, \ldots, x_{m}\right) ; s_{1}, \ldots, s_{m}\right\}=\int_{0}^{\infty} \ldots \int_{0}^{\infty} x_{1}^{s_{1}-1} \ldots x_{m}^{s_{m}-1} f\left(x_{1}, \ldots, x_{m}\right) d x_{1} \ldots d x_{m}
$$

provided that the multiple integral exists.

In particular, if $f\left(x_{1}, \ldots, x_{m}\right)=\prod_{i=1}^{m} f_{i}\left(x_{i}\right)$, then

$$
M\left\{f\left(x_{1}, \ldots, x_{m}\right) ; s_{1}, \ldots, s_{m}\right\}=\prod_{i=1}^{m} M_{1}\left\{f_{i}\left(x_{i}\right) ; s_{i}\right\},
$$

where $M_{1}$ is the one-dimensional Mellin transform.

Also the multidimensional Mellin convolution of two functions $f\left(x_{1}, \ldots, x_{m}\right)$ and $g\left(x_{1}, \ldots, x_{m}\right)$ is defined by

$$
(f * g)\left(x_{1}, \ldots, x_{m}\right)=\int_{0}^{\infty} \ldots \int_{0}^{\infty} y_{1}^{-1} \ldots y_{m}^{-1} f\left(\frac{x_{1}}{y_{1}}, \ldots, \frac{x_{m}}{y_{m}}\right) g\left(y_{1}, \ldots, y_{m}\right) d y_{1} \ldots d y_{m}
$$


provided that the multiple integral exists.

\section{Multidimensional Mellin transform of $u\left(x_{1}, \ldots, x_{m}\right)$}

In order to solve the multidimensional integral equation (1.4), the following result is needed.

Lemma: Let $U\left(s_{1}, \ldots, s_{m}\right)=M\left\{u\left(x_{1}, \ldots, x_{m}\right) ; s_{1}, \ldots, s_{m}\right\}$, where $u\left(x_{1}, \ldots, x_{m}\right)$ is defined by (1.5), then

$$
\begin{aligned}
& U\left(s_{1}, \ldots, s_{m}\right) \\
= & \prod_{i=1}^{m}\left\{\sum_{e_{i}=0}^{n_{i}} \sum_{p_{i}=0}^{\alpha_{i}+q_{i} n_{i}} \frac{\left(-n_{i}\right)_{e_{i}}\left(-\alpha_{i}-q_{i} n_{i}\right) p_{p_{i}}}{e_{i} ! p_{i} !}(-1)^{p_{i}} k_{i}^{n_{i}-e_{i}} A_{i}^{p_{i}}\right. \\
& \times B_{i}^{\alpha_{i}+q_{i} n_{i}-p_{i}} l_{i}^{e_{i}} \frac{\left(-\tau_{i}\right)^{-\left(s_{i}+l_{i} e_{i}+p_{i}\right) / r_{i}}}{\left|r_{i}\right|} \Gamma\left(\frac{s_{i}+l_{i} e_{i}+p_{i}}{r_{i}}\right)\left(-\frac{s_{i}+l_{i} e_{i}+p_{i}}{l_{i}}\right)_{e_{i}} \\
& \left.\times \Gamma\left(\frac{-\beta_{i}}{\tau_{i}}-c_{i} n_{i}-\frac{s_{i}+l_{i} e_{i}+p_{i}}{r_{i}}\right)\left\{\Gamma\left(\frac{-\beta_{i}}{\tau_{i}}-c_{i} n_{i}\right)\right\}^{-1}\right\}
\end{aligned}
$$

provided that $\left|\arg \tau_{i}<\pi\right|, 0<\operatorname{Re}\left(s_{i}+l_{i} e_{i}+p_{i}\right)<r_{i} \operatorname{Re}\left(\frac{-\beta_{i}}{\tau_{i}}-c_{i} n_{i}\right)$, when $r_{i}>0$; $r_{i} \operatorname{Re}\left(\frac{-\beta_{i}}{\tau_{i}}-c_{i} n_{i}\right)<\operatorname{Re}\left(s_{i}+l_{i} e_{i}+p_{i}\right)<0$ when $r_{i}<0$, for $(i=1, \ldots, m), m \in N_{0}$.

Proof. Making use of the binomial expansion for $\left[x_{i}^{l_{i}}\left(k_{i}+x_{i} D x_{i}\right)\right]^{n_{i}}$, we find that

$$
\begin{aligned}
& \quad u\left(x_{1}, \ldots, x_{m}\right) \\
& =\prod_{i=1}^{m}\left\{\sum_{e_{i}=0}^{n_{i}} \sum_{p_{i}=0}^{\alpha_{i}+q_{i} n_{i}} \frac{\left(-n_{i}\right)_{e_{i}}\left(-\alpha_{i}-q_{i} n_{i}\right)_{p_{i}}}{e_{i} ! p_{i} !}(-1)^{e_{i}+p_{i}} k_{i}^{n_{i}-e_{i}} A_{i}^{p_{i}}\right. \\
& \left.\quad \times B_{i}^{\alpha_{i}+q_{i} n_{i}-p_{i}} x_{i}^{l_{i}\left(n_{i}-e_{i}\right)}\left(x_{i}^{l_{i}+1} D x_{i}\right)^{e_{i}}\left[x_{i}^{p_{i}}\left\{1-\frac{\tau_{i}}{x_{i}^{-r_{i}}}\right\}^{\frac{\beta_{i}}{\tau_{i}}+c_{i} n_{i}}\right]\right\} .
\end{aligned}
$$

Applying the Multidimensional Mellin transform to both sides of (2.2), making use of (1.7) and applying the following known formulas [15, p.14, eq. (23); 1, p. 307 , eq. (7)]

$$
M_{1}\left\{\left(x_{i}^{l_{i}+1} D x_{i}\right)^{n_{i}} f\left(x_{i}\right) ; s_{i}\right\}=l_{i}^{n_{i}}\left(-\frac{s_{i}+l_{i} n_{i}}{l_{i}}\right)_{n_{i}} M_{1}\left\{f\left(x_{i}\right) ; s_{i}+l_{i} n_{i}\right\}
$$

and

$$
M_{1}\left\{x_{i}^{\mu_{i}} f\left(x_{i}\right) ; s_{i}\right\}=M_{1}\left\{f\left(x_{i}\right) ; s_{i}+\mu_{i}\right\}
$$


provided that the Mellin transforms of (2.3) and (2.4) exist, we get

$$
\begin{aligned}
& u\left(x_{1}, \ldots, x_{m}\right) \\
= & \prod_{i=1}^{m}\left\{\sum_{e_{i}=0}^{n_{i}} \sum_{p_{i}=0}^{\alpha_{i}+q_{i} n_{i}} \frac{\left(-n_{i}\right)_{e_{i}}\left(-\alpha_{i}-q_{i} n_{i}\right)_{p_{i}}}{e_{i} ! p_{i} !}(-1)^{e_{i}+p_{i}} k_{i}^{n_{i}-e_{i}} A_{i}^{p_{i}}\right. \\
& \left.\times B_{i}^{\alpha_{i}+q_{i} n_{i}-p_{i}} l_{i}^{e_{i}}\left(-\frac{s_{i}+l_{i} e_{i}}{l_{i}}\right) e_{i} M_{1}\left[\left\{1-\frac{\tau_{i}}{x_{i}^{-r_{i}}}\right\}^{\frac{\beta_{i}}{\tau_{i}}+c_{i} n_{i}} ; s_{i}+l_{i} e_{i}+p_{i}\right]\right\} .
\end{aligned}
$$

Again making use of (2.4) and the following result [3, p. 311, eq. (30)],

$$
M_{1}\left\{\left(1+a_{i} x_{i}^{r_{i}}\right)^{-\nu_{i}} ; s_{i}\right\}=\frac{1}{\left|r_{i}\right|} a_{i}^{\frac{-s_{i}}{r_{i}}} \frac{\Gamma\left(\frac{s_{i}}{r_{i}}\right) \Gamma\left(\nu_{i}-\frac{s_{i}}{r_{i}}\right)}{\Gamma\left(\nu_{i}\right)},
$$

where $\left|\operatorname{arga}_{i}\right|<\pi, 0<\operatorname{Res}_{i}<r_{i} \operatorname{Re\nu }_{i}$, when $r_{i}>0 ; r_{i} \operatorname{Re\nu }_{i}<\operatorname{Res}_{i}<0$, when $r_{i}<0$, for $(i=1, \ldots, m), m \in N_{0}$. The result readily follows.

\section{Solution of the integral equation (1.4)}

Theorem: Let the multidimensional Mellin transforms $F\left(s_{1}, \ldots, s_{m}\right), G\left(s_{1}, \ldots, s_{m}\right)$ and $U\left(s_{1}, \ldots, s_{m}\right)$ of the functions $f\left(x_{1}, \ldots, x_{m}\right), g\left(x_{1}, \ldots, x_{m}\right)$ and $u\left(x_{1}, \ldots, x_{m}\right)$ defined by (1.5) exist and be analytic in some infinite strip $\zeta_{i}<\operatorname{Res}_{i}<\eta_{i}$ of the complex s-plane. Also suppose that for a fixed $\gamma_{i} \in\left(\zeta_{i}, \eta_{i}\right), \bar{u}$ is defined by

$$
\begin{aligned}
\bar{u}\left(x_{1}, \ldots, x_{m}\right) & =M^{-1}\left\{\bar{U}\left(s_{1}, \ldots, s_{m}\right) ; x_{1}, \ldots, x_{m}\right\} \\
& =\frac{1}{(2 \pi i)^{m}} \int_{\gamma_{1}-i \infty}^{\gamma_{1}+i \infty} \ldots \int_{\gamma_{m}-i \infty}^{\gamma_{m}+i \infty} x_{1}^{s_{1}} \ldots x_{m}^{s_{m}} \bar{U}\left(s_{1}, \ldots, s_{m}\right) d s_{1} \ldots d s_{m},
\end{aligned}
$$

where

$$
\begin{aligned}
& \bar{U}\left(s_{1}, \ldots, s_{m}\right) \\
= & \prod_{i=1}^{m}\left[\mu_{i}^{l_{i}} \frac{\Gamma\left(-\frac{s_{i}}{\mu_{i}}\right)}{\Gamma\left(-l_{i}-\frac{s_{i}}{\mu_{i}}\right)} \sum_{e_{i}=0}^{n_{i}} \sum_{p_{i}=0}^{\alpha_{i}+q_{i} n_{i}} \frac{\left(-n_{i}\right) e_{e_{i}}\left(-\alpha_{i}-q_{i} n_{i}\right)_{p_{i}}}{e_{i} ! p_{i} !}(-1)^{p_{i}} k_{i}^{n_{i}-e_{i}}\right. \\
& A_{i}^{p_{i}} B_{i}^{\alpha_{i}+q_{i} n_{i}-p_{i}} l_{i}^{e_{i}} \frac{\left(-\tau_{i}\right)^{-\left(s_{i}+\mu_{i} l_{i}+\lambda_{i}+l_{i} e_{i}+p_{i}\right) / r_{i}}}{\left|r_{i}\right|} \Gamma\left(1+\frac{s_{i}+\mu_{i} l_{i}+\lambda_{i}+l_{i} e_{i}}{l_{i}}\right) \\
& \left.\frac{\Gamma\left(\frac{s_{i}+\mu_{i} l_{i}+\lambda_{i}+l_{i} e_{i}+p_{i}}{r_{i}}\right) \Gamma\left(\frac{-\beta_{i}}{\tau_{i}}-c_{i} n_{i}-\frac{s_{i}+\mu_{i} l_{i}+\lambda_{i}+l_{i} e_{i}+p_{i}}{r_{i}}\right)}{\Gamma\left(1+\frac{s_{i}+\mu_{i} l_{i}+\lambda_{i}}{l_{i}}\right) \Gamma\left(\frac{-\beta_{i}}{\tau_{i}}-c_{i} n_{i}\right)}\right]^{-1}
\end{aligned}
$$

provided that $\left|\arg \tau_{i}<\pi\right|, 0<\operatorname{Re}\left(s_{i}+\mu_{i} l_{i}+\lambda_{i}+l_{i} e_{i}+p_{i}\right)<r_{i} \operatorname{Re}\left(\frac{-\beta_{i}}{\tau_{i}}-c_{i} n_{i}\right)$, when $r_{i}>0 ; r_{i} \operatorname{Re}\left(\frac{-\beta_{i}}{\tau_{i}}-c_{i} n_{i}\right)<\operatorname{Re}\left(s_{i}+\mu_{i} l_{i}+\lambda_{i}+l_{i} e_{i}+p_{i}\right)<0$ when $r_{i}<0$, for 
$(i=1, \ldots, m), m \in N_{0}$. Then the integral equation (1.4) has its solution given by

$$
\begin{aligned}
& f\left(x_{1}, \ldots, x_{m}\right) \\
= & x_{1}^{-\mu_{1} l_{1}-\lambda_{1}} \ldots x_{m}^{-\mu_{m} l_{m}-\lambda_{m}} \int_{0}^{\infty} \ldots \int_{0}^{\infty} y_{1}^{-1} \ldots y_{m}^{-1} \bar{u}\left(\frac{x_{1}}{y_{1}}, \ldots, \frac{x_{m}}{y_{m}}\right) \\
& \left(y_{1}^{\mu_{1}+1} D y_{1}\right)^{l_{1}} \ldots\left(y_{m}^{\mu_{m}+1} D y_{m}\right)^{l_{m}}\left\{y_{1}^{\lambda_{1}} \ldots y_{m}^{\lambda_{m}} g\left(y_{1}, \ldots, y_{m}\right)\right\} d y_{1} \ldots d y_{m}
\end{aligned}
$$

provided that the integral exist.

Proof. Making use of (1.8), then (1.4) can be written as

$$
(U * F)\left(x_{1}, \ldots, x_{m}\right)=g\left(x_{1}, \ldots, x_{m}\right) .
$$

Now, multidimensional Mellin transform of (3.4) yields

$$
U\left(s_{1}, \ldots, s_{m}\right) F\left(s_{1}, \ldots, s_{m}\right)=G\left(s_{1}, \ldots, s_{m}\right),
$$

where $U\left(s_{1}, \ldots, s_{m}\right)$ is given by $(2.1)$.

Replacing $s_{i}$ by $s_{i}+\mu_{i} l_{i}+\lambda_{i}$, we obtain

$$
\begin{aligned}
& F\left(s_{1}+\mu_{1} l_{1}+\lambda_{1}, \ldots, s_{m}+\mu_{m} l_{m}+\lambda_{m}\right) \\
= & \bar{U}\left(s_{1}, \ldots, s_{m}\right) \times M\left\{\left(x_{1}^{\mu_{1}+1} D x_{1}\right)^{l_{1}} \ldots\left(x_{m}^{\mu_{m}+1} D x_{m}\right)^{l_{m}}\left\{x_{1}^{\lambda_{1}} \ldots x_{m}^{\lambda_{m}} g\left(x_{1}, \ldots, x_{m}\right)\right\}\right\}
\end{aligned}
$$

Again using the multidimensional Mellin convolution theorem and (2.4), we find that

$$
\begin{aligned}
& M\left\{x_{1}^{\mu_{1} l_{1}+\lambda_{1}} \ldots x_{m}^{\mu_{m} l_{m}+\lambda_{m}} f\left(x_{1}, \ldots, x_{m}\right) ; s_{1}, \ldots, s_{m}\right\} \\
= & M\left\{\int_{0}^{\infty} \ldots \int_{0}^{\infty} y_{1}^{-1} \ldots y_{m}^{-1} \bar{u}\left(\frac{x_{1}}{y_{1}}, \ldots, \frac{x_{m}}{y_{m}}\right)\left\{y_{1}^{-1} \ldots y_{m}^{-1} g\left(y_{1}, \ldots, y_{m}\right)\right\}\right. \\
& \left.\left(y_{1}^{\mu_{1}+1} D y_{1}\right)^{l_{1}} \ldots\left(y_{m}^{\mu_{m}+1} D y_{m}\right)^{l_{m}} d y_{1} \ldots d y_{m} ; s_{1}, \ldots, s_{m}\right\}
\end{aligned}
$$

Inverting both sides of (3.7) by using the Mellin inversion theorem [3, p. 307, eq. (1)], we arrive at the required solution (3.3).

\section{Applications}

Since the generalized polynomial set defined by (1.3) has a large number of special cases, one can obtain the solutions of a number of multidimensional integral equations of the type (1.4) with the kernels involving products of Laguerre polynomials, Hermite polynomials, Bessel polynomials, $H_{n}^{(r)}(x, \alpha, \beta)$ polynomials defined by Gould and Hopper [4], $F_{n}^{(r)}(x, \alpha, q, \beta)$ polynomials defined by Chatterjea 
[2], $G_{n}^{(r)}(x, \alpha, \beta, l)$ polynomials defined by Srivastava and Singhal [14], and several other polynomials. We mention in what follows some of these special cases.

If we put $A_{i}=1, B_{i}=0$ and $k_{i}=0$, then we get the following result contained in the following corollary.

Corollary 2: Under the hypothesis of Theorem, the multidimensional integral equation

$$
\int_{0}^{\infty} \ldots \int_{0}^{\infty} y_{1}^{-1} \ldots y_{m}^{-1} u_{2}\left(\frac{x_{1}}{y_{1}}, \ldots, \frac{x_{m}}{y_{m}}\right) f\left(y_{1}, \ldots, y_{m}\right) d y_{1} \ldots d y_{m}=g\left(x_{1}, \ldots, x_{m}\right)
$$

where $x_{i}>0, \forall i=1, \ldots, m$, and

$$
\begin{aligned}
u_{2}\left(x_{1}, \ldots, x_{m}\right) & =\prod_{i=1}^{m} x_{i}^{\alpha_{i}}\left(1-\tau_{i} x_{i}^{r_{i}}\right)^{\beta_{i} / \tau_{i}} S_{n_{i}}^{\alpha_{i}, \beta_{i}, \tau_{i}}\left[x_{i} ; r_{i}, c_{i}, q_{i}, 1,0,0, l_{i}\right] \\
& =\prod_{i=1}^{m}\left(x_{i}^{l_{i}+1} D_{x_{i}}\right)^{n_{i}}\left\{x_{i}^{\alpha_{i}+q_{i} n_{i}}\left(1-\tau_{i} x_{i}^{r_{i}}\right)^{\beta_{i} / \tau_{i}+c_{i} n_{i}}\right\}
\end{aligned}
$$

has its solution given by

$$
\begin{array}{r}
f\left(x_{1}, \ldots, x_{m}\right)=x_{1}^{-\mu_{1} l_{1}-\lambda_{1}} \ldots x_{m}^{-\mu_{m} l_{m}-\lambda_{m}} \int_{0}^{\infty} \ldots \int_{0}^{\infty} y_{1}^{-1} \ldots y_{m}^{-1} \overline{u_{2}}\left(\frac{x_{1}}{y_{1}}, \ldots, \frac{x_{m}}{y_{m}}\right) \\
\left(y_{1}^{\mu_{1}+1} D y_{1}\right)^{l_{1}} \ldots\left(y_{m}^{\mu_{m}+1} D y_{m}\right)^{l_{m}}\left\{y_{1}^{\lambda_{1}} \ldots y_{m}^{\lambda_{m}} g\left(y_{1}, \ldots, y_{m}\right)\right\} d y_{1} \ldots d y_{m}
\end{array}
$$

provided the integral exist. where $\overline{u_{2}}\left(x_{1}, \ldots, x_{m}\right)$ is the the multidimensional Mellin inverse transform of

$$
\begin{aligned}
& \bar{U}_{2}\left(s_{1}, \ldots, s_{m}\right) \\
= & \prod_{i=1}^{m}\left[\frac{\left|r_{i}\right|}{\mu_{i}^{l_{i}} l_{i}^{n_{i}}}\left(-\tau_{i}\right)^{-\left(s_{i}+\mu_{i} l_{i}+n_{i}\left(l_{i}+q_{i}\right)+\lambda_{i}+\alpha_{i}\right) / r_{i}} \frac{\Gamma\left(\frac{-\beta_{i}}{\tau_{i}}-c_{i} n_{i}\right) \Gamma\left(-l_{i}-\frac{s_{i}}{\mu_{i}}\right)}{\Gamma\left(-\frac{s_{i}}{\mu_{i}}\right) \Gamma\left(\frac{-s_{i}-\mu_{i} l_{i}+\lambda_{i}}{l_{i}}\right)}\right. \\
& \left.\frac{\Gamma\left(-n_{i}-\frac{s_{i}+\mu_{i} l_{i}+\lambda_{i}}{l_{i}}\right)}{\Gamma\left(\frac{s_{i}+\mu_{i} l_{i}+\lambda_{i}+n_{i}\left(l_{i}+q_{i}\right)+\alpha_{i}}{r_{i}}\right) \Gamma\left(-c_{i} n_{i}-\frac{\beta_{i}}{\tau_{i}}-\frac{s_{i}+\mu_{i} l_{i}+\lambda_{i}+n_{i}\left(l_{i}+q_{i}\right)+\alpha_{i}}{r_{i}}\right)}\right],
\end{aligned}
$$

provided that $\left|\arg \tau_{i}<\pi\right|, 0<\operatorname{Re}\left(s_{i}+\mu_{i} l_{i}+\lambda_{i}+n_{i}\left(l_{i}+q_{i}\right)+\alpha_{i}\right)<$ $r_{i} \operatorname{Re}\left(\frac{-\beta_{i}}{\tau_{i}}-c_{i} n_{i}\right)$, when $r_{i}>0 ; r_{i} \operatorname{Re}\left(\frac{-\beta_{i}}{\tau_{i}}-c_{i} n_{i}\right)<\operatorname{Re}\left(s_{i}+\mu_{i} l_{i}+\lambda_{i}+n_{i}\left(l_{i}+\right.\right.$ $\left.\left.q_{i}\right)+\alpha_{i}\right)<0$ when $r_{i}<0$, for $(i=1, \ldots, m), m \in N_{0}$. 
Now, applying the Mellin inversion formula (3.1) and replacing $s_{i}$ by $-s_{i} \mathrm{w}$ get

$$
\begin{aligned}
\bar{u}\left(x_{1}, \ldots, x_{m}\right)= & \prod_{i=1}^{m}\left[\frac{\left|r_{i}\right|}{\mu_{i}^{l_{i}} l_{i}^{n_{i}}}\left(-\tau_{i}\right)^{\left(\mu_{i} l_{i}+n_{i}\left(l_{i}+q_{i}\right)+\lambda_{i}+\alpha_{i}\right) / r_{i}}\right. \\
& \Gamma\left(\frac{-\beta_{i}}{\tau_{i}}-c_{i} n_{i}\right) \frac{1}{(2 \pi i)^{m}} \int_{\gamma_{1}-i \infty}^{\gamma_{1}+i \infty} \ldots \int_{-i \infty}^{\gamma_{m}+i \infty} \varphi_{1}\left(s_{1}\right) \ldots \varphi_{m}\left(s_{m}\right) \\
& \left.\left(\frac{x_{1}}{\left(-\tau_{1}\right)^{1 / r_{1}}}\right)^{s_{1}} \ldots\left(\frac{x_{m}}{\left(-\tau_{m}\right)^{1 / r_{m}}}\right)^{s_{m}} d s_{1} \ldots d s_{m}\right]
\end{aligned}
$$

where

$$
\begin{aligned}
\varphi_{i}\left(s_{i}\right)= & \frac{\Gamma\left(-l_{i}+\frac{s_{i}}{\mu_{i}}\right) \Gamma\left(-n_{i}-\frac{-s_{i}+\mu_{i} l_{i}+\lambda_{i}}{l_{i}}\right)}{\Gamma\left(\frac{s_{i}}{\mu_{i}}\right) \Gamma\left(\frac{s_{i}-\mu_{i} l_{i}+\lambda_{i}}{l_{i}}\right) \Gamma\left(\frac{-s_{i}+\mu_{i} l_{i}+\lambda_{i}+n_{i}\left(l_{i}+q_{i}\right)+\alpha_{i}}{r_{i}}\right)} \\
& \frac{1}{\Gamma\left(-c_{i} n_{i}-\frac{\beta_{i}}{\tau_{i}}-\frac{-s_{i}+\mu_{i} l_{i}+\lambda_{i}+n_{i}\left(l_{i}+q_{i}\right)+\alpha_{i}}{r_{i}}\right)} .
\end{aligned}
$$

Under various restrictions on the non-zero constants $r_{i}, l_{i}, \mu_{i}, i=1,2, \ldots, m$, the contour integral in (4.5) can be expressed in terms of multivariable $\mathrm{H}$-functions or a product of $m$ Foxe's H-functions (see Srivastava et al. [10, ch.2]). For example, the solution of the the multidimensional integral equation (4.1) with the kernel defined by (4.2) can be written as

(4.7) $f\left(x_{1}, \ldots, x_{m}\right)$

$$
\begin{aligned}
& =\prod_{i=1}^{m}\left[\frac{\left|r_{i}\right|}{\mu_{i}^{l_{i}} l_{i}^{n_{i}}}\left(-\tau_{i}\right)^{\left(\mu_{i} l_{i}+n_{i}\left(l_{i}+q_{i}\right)+\lambda_{i}+\alpha_{i}\right) / r_{i}} \Gamma\left(\frac{-\beta_{i}}{\tau_{i}}-c_{i} n_{i}\right) x_{i}^{-\mu_{i} l_{i}-\lambda_{i}}\right] \\
& \int_{0}^{\infty} \ldots \int_{0}^{\infty} y_{1}^{-1} \ldots y_{m}^{-1}\left(y_{1}^{\mu_{1}+1} D y_{1}\right)^{l_{1}} \ldots\left(y_{m}^{\mu_{m}+1} D y_{m}\right)^{l_{m}}\left\{y_{1}^{\lambda_{1}} \ldots y_{m}^{\lambda_{m}} g\left(y_{1}, \ldots, y_{m}\right)\right\} \\
& H_{0,0 ; 3,3 ; \ldots ; 3,3}^{0,0 ; 1, \ldots ; 1,1}\left[\frac{x_{1}}{y_{1}\left(-\tau_{1}\right)^{1 / r_{1}}}, \ldots, \frac{x_{m}}{y_{m}\left(-\tau_{m}\right)^{1 / r_{m}}} \mid \begin{array}{l}
: \\
: \\
\left(-l_{1},-1 / \mu_{1}\right),\left(1+\frac{\mu_{1} l_{1}+\lambda_{1}}{l_{1}}, 1 / l_{1}\right),
\end{array}\right. \\
& \left(\frac{\mu_{1} l_{1}+\lambda_{1}+n_{1}\left(l_{1}+q_{1}\right)+\alpha_{1}}{r_{1}}, 1 / r_{1}\right) ; \ldots ;\left(1+n_{m}+\frac{\mu_{m} l_{m}+\lambda_{m}}{l_{m}}, 1 / l_{m}\right),\left(0,-1 / \mu_{m}\right), \\
& \left(1+\frac{\beta_{1}}{\tau_{1}}+c_{1} n_{1}+\frac{\mu_{1} l_{1}+\lambda_{1}+n_{1}\left(l_{1}+q_{1}\right)+\alpha_{1}}{r_{1}}, 1 / r_{1}\right) ; \ldots ;\left(-l_{m},-1 / \mu_{m}\right),\left(1+\frac{\mu_{m} l_{m}+\lambda_{m}}{l_{m}}, 1 / l_{m}\right), \\
& \left.\begin{array}{l}
\left(\frac{\mu_{m} l_{m}+\lambda_{m}+n_{m}\left(l_{m}+q_{m}\right)+\alpha_{m}}{r_{m}}, 1 / r_{m}\right) \\
\left(1+\frac{\beta_{m}}{\tau_{m}}+c_{m} n_{m}+\frac{\mu_{m} l_{m}+\lambda_{m}+n_{m}\left(l_{m}+q_{m}\right)+\alpha_{m}}{r_{m}}, 1 / r_{m}\right)
\end{array}\right] d y_{1} \ldots d y_{m},
\end{aligned}
$$

for $r_{i}>0, \mu_{i}<0, l_{i}>0(i=1,2, . ., m)$. 
Also, on setting $A_{i}=1, B_{i}=0, k_{i}=0$ as $\tau_{i} \rightarrow 0$, we get

$$
S_{n_{i}}^{\left(\alpha_{i}+l_{i} n_{i}\right), \beta_{i}, 0}\left[x_{i} ; r_{i},-l_{i}, 1,0,0, l_{i}\right]=n_{i} ! G_{n_{i}}^{\left(\alpha_{i}\right)}\left(x_{i}, r_{i}, \beta_{i}, l_{i}\right)
$$

where $G_{n}^{\alpha}(x, r, \beta, l)$ is the class of polynomials studied by Srivastava and Singhal [14], so we have the following Corollary.

Corollary 4: Under the hypothesis of Theorem, the multidimensional integral equation

$$
\int_{0}^{\infty} \ldots \int_{0}^{\infty} y_{1}^{-1} \ldots y_{m}^{-1} u_{4}\left(\frac{x_{1}}{y_{1}}, \ldots, \frac{x_{m}}{y_{m}}\right) f\left(y_{1}, \ldots, y_{m}\right) d y_{1} \ldots d y_{m}=g\left(x_{1}, \ldots, x_{m}\right)
$$

where $x_{i}>0, \forall i=1, \ldots, m$, and

$$
\begin{aligned}
u_{4}\left(x_{1}, \ldots, x_{m}\right) & =\prod_{i=1}^{m} n_{i} ! x_{i}^{\alpha_{i}+l_{i} n_{i}} \exp \left(-\beta_{i} x_{i}^{r_{i}}\right) G_{n_{i}}^{\left(\alpha_{i}\right)}\left(x_{i}, r_{i}, \beta_{i}, l_{i}\right) \\
& =\prod_{i=1}^{m}\left(x_{i}^{l_{i}+1} D_{x_{i}}\right)^{n_{i}}\left\{x_{i}^{\alpha_{i}} \exp \left(-\beta_{i} x_{i}^{r_{i}}\right)\right\},
\end{aligned}
$$

has its solution given by

$$
\begin{gathered}
f\left(x_{1}, \ldots, x_{m}\right)=x_{1}^{-\mu_{1} l_{1}-\lambda_{1}} \ldots x_{m}^{-\mu_{m} l_{m}-\lambda_{m}} \int_{0}^{\infty} \ldots \int_{0}^{\infty} y_{1}^{-1} \ldots y_{m}^{-1} \overline{u_{4}}\left(\frac{x_{1}}{y_{1}}, \ldots, \frac{x_{m}}{y_{m}}\right) \\
\left(y_{1}^{\mu_{1}+1} D y_{1}\right)^{l_{1}} \ldots\left(y_{m}^{\mu_{m}+1} D y_{m}\right)^{l_{m}}\left\{y_{1}^{\lambda_{1}} \ldots y_{m}^{\lambda_{m}} g\left(y_{1}, \ldots, y_{m}\right)\right\} d y_{1} \ldots d y_{m}
\end{gathered}
$$

provided the integral exist, and $\bar{u}_{4}\left(x_{1}, \ldots, x_{m}\right)$ is the the multidimensional Mellin inverse transform of

$$
\begin{aligned}
\bar{U}_{4}\left(s_{1}, \ldots, s_{m}\right)= & \prod_{i=1}^{m}\left[\frac{\left|r_{i}\right|}{\mu_{i}^{l_{i}} l_{i}^{n_{i}}}\left(\beta_{i}\right)^{\left(s_{i}+\mu_{i} l_{i}+n_{i} l_{i}+\lambda_{i}+\alpha_{i}\right) / r_{i}}\right. \\
& \left.\frac{\Gamma\left(-l_{i}-\frac{s_{i}}{\mu_{i}}\right) \Gamma\left(-n_{i}-\frac{s_{i}+\mu_{i} l_{i}+\lambda_{i}}{l_{i}}\right)}{\Gamma\left(\frac{s_{i}+\mu_{i} l_{i}+\lambda_{i}+n_{i} l_{i}+\alpha_{i}}{r_{i}}\right) \Gamma\left(-\frac{s_{i}}{\mu_{i}}\right) \Gamma\left(-\frac{s_{i}+\mu_{i} l_{i}+\lambda_{i}}{l_{i}}\right)}\right],
\end{aligned}
$$

provided that $\operatorname{Re}\left(s_{i}+\mu_{i} l_{i}+\lambda_{i}+n_{i} l_{i}+\alpha_{i}\right)>0$, when $r_{i}>0 ; \operatorname{Re}\left(s_{i}+\mu_{i} l_{i}+\lambda_{i}+\right.$ $\left.n_{i} l_{i}+\alpha_{i}\right)<0$, when $r_{i}<0$, for $(i=1, \ldots, m), m \in N_{0}$.

Now, applying the Mellin inversion formula (3.1) in (4.12) and replacing $s_{i}$ by 
$-s_{i}$ w get

$$
\begin{aligned}
& \bar{U}_{4}\left(s_{1}, \ldots, s_{m}\right) \\
= & \prod_{i=1}^{m}\left[\frac{\left|r_{i}\right|}{\mu_{i}^{l_{i}} l_{i}^{n_{i}}}(\beta i)^{\left(\mu_{i} l_{i}+n_{i} l_{i}+\lambda_{i}+\alpha_{i}\right) / r_{i}} \frac{1}{(2 \pi i)^{m}} \int_{\gamma_{1}-i \infty}^{\gamma_{1}+i \infty} \ldots \int_{\gamma_{m}-i \infty}^{\gamma_{m}+i \infty}\right. \\
& \left.\varphi_{1}\left(s_{1}\right) \ldots \varphi_{m}\left(s_{m}\right)\left(\frac{x_{1}}{\left(\beta_{1}\right)^{1 / r_{1}}}\right)^{s_{1}} \ldots\left(\frac{x_{m}}{\left(\beta_{m}\right)^{1 / r_{m}}}\right)^{s_{m}} d s_{1} \ldots d s_{m}\right],
\end{aligned}
$$

where

$$
\varphi_{i}\left(s_{i}\right)=\frac{\Gamma\left(-l_{i}+\frac{s_{i}}{\mu_{i}}\right) \Gamma\left(-n_{i}-\frac{-s_{i}+\mu_{i} l_{i}+\lambda_{i}}{l_{i}}\right)}{\Gamma\left(\frac{s_{i}}{\mu_{i}}\right) \Gamma\left(\frac{s_{i}-\mu_{i} l_{i}+\lambda_{i}}{l_{i}}\right) \Gamma\left(\frac{-s_{i}+\mu_{i} l_{i}+\lambda_{i}+n_{i} l_{i}+\alpha_{i}}{r_{i}}\right)} .
$$

In particular, if $\lambda_{i}>0, \mu_{i}>0, r_{i}>0$, then the solution (4.11) can be written as

(4.15) $f\left(x_{1}, \ldots, x_{m}\right)$

$$
\begin{aligned}
& =\prod_{i=1}^{m}\left(\frac{\left|r_{i}\right|}{\mu_{i}^{l_{i}} l_{i}^{n_{i}}}\left(\beta_{i}\right)^{\left(\mu_{i} l_{i}+n_{i} l_{i}+\lambda_{i}+\alpha_{i}\right) / r_{i}} x_{i}^{-\mu_{i} l_{i}-\lambda_{i}}\right) \\
& \int_{0}^{\infty} \ldots \int_{0}^{\infty} H_{0,0 ; 3,2 ; \ldots ; 3,2}^{0,0 ; 0,2 ; \ldots ; 0,2}\left[\frac{x_{1}}{y_{1}\left(\beta_{1}\right)^{1 / r_{1}}}, \ldots, \frac{x_{m}}{y_{m}\left(\beta_{m}\right)^{1 / r_{m}}} \mid \begin{array}{l}
:\left(1+n_{1}+\frac{\mu_{1} l_{1}+\lambda_{1}}{l_{1}}, 1 / l_{1}\right),\left(1+l_{1}, 1 / \mu_{1}\right), \\
-\left(1,1 / \mu_{1}\right),\left(1+\frac{\mu_{1} l_{1}+\lambda_{1}}{l_{1}}, 1 / l_{1}\right)
\end{array}\right. \\
& \left.\begin{array}{c}
\left(\frac{\mu_{1} l_{1}+\lambda_{1}+n_{1} l_{1}+\alpha_{1}}{r_{1}}, 1 / r_{1}\right) ; \ldots ;\left(1+n_{m}+\frac{\mu_{m} l_{m}+\lambda_{m}}{l_{m}}, 1 / l_{m}\right),\left(1+l_{m}, 1 / \mu_{m}\right),\left(\frac{\mu_{m} l_{m}+\lambda_{m}+n_{m} l_{m}+\alpha_{m}}{r_{m}}, 1 / r_{m}\right) \\
; \ldots ;\left(1,1 / \mu_{m}\right),\left(1+\frac{\mu_{m} l_{m}+\lambda_{m}}{l_{m}}, 1 / l_{m}\right)
\end{array}\right]
\end{aligned}
$$

$d y_{1} \ldots d y_{m}$

If we set $m=1$ in (4.15), we get the result obtained by Srivastava [15] and by setting $A_{1}=0, B_{1}=0, k_{1}=q_{1}=0, l_{1}=\mu_{1}=-1, m=1$, and $\tau_{1} \rightarrow 0$ in (1.6), we get the solution of the integral equation considered by Lala and Shrivastava $[6,7]$.

Acknowledgement The authors wish to thank the anonymous referee for valuable comments and suggestions.

\section{References}

[1] Agrawal B. D. and Chaubey J. P., Operational derivation of generating relations for generalized polynomials, Indian J. Pure Appl. Math., 11(1980), 1155-1157; ibid. 11(1981), 357-379. 
[2] Chatterjea S. K., Quelques fonctions génératrics des Poylnomes d'Hermite du point de vue de algebre de Lie, C. R. Acad. Sci. Paris, Ser., A-B268(1969), 600-602.

[3] Erdélyi A., Magnus W., Oberhettinger F. and Tricomi F. G., Tables of integral transforms Vol. I, McGraw-Hill, New York, 1954.

[4] Gould H. W. and Hopper A. T., Operational formulas connected with two generalizations of Hermite polynomials, Duke Math. J., 29(1962), 51-63.

[5] Krall H. L. and Frink O. A., A new class of orthogonal polynomials; Bessel polynomials, Trans. Am. Math. Soc., 65(1949), 100-115.

[6] Lala A.and Shrivastava P. N., Inversion of an integral involving a generalized Hermite polynomial, Indian J. Pure Appl. Math., 21(1990), 163-166.

[7] Lala A. and Shrivastava P. N., Inversion of an integral involving a generalized function, Bull. Calcutta Math. Soc., 82(1990), 115-118.

[8] Raizad S. K., A study of unified representation of special functions of mathematical physics and their use in statistical and boundary value problems, $\mathrm{Ph} . \mathrm{D}$. thesis (Bundelkhand Univ., Jhansi, India), 1991.

[9] Saigo M., Goyal S. P. and Sexena S. A., A theorem relating a generalized Weyl fractional integral, Laplace and Varma transforms with applications, J. Fract. calc., 13(1998), 43-56.

[10] Srivastava H. M., Gupta K. C. and Goyal S. P., The H-Functions of One and Two Variables with Applications, South Asian Publ., New Delhi-Madras, 1982.

[11] Srivastava H. M. and Monocha H. L., A Treatise on Generating Functions, Wiley/Halsted, New York, 1984

[12] Srivastava H. M. and Panda R., Certain multidimensional integral transformation I and II, Nederl. Akad. Wetensch. Indag Math., 40(1978), 118-131, 132-144.

[13] Srivastava H. M. and Panda R., On the unified presentation of certain classical polynomials, Boll. Un. Mat. Ital., 12(4)(1975), 306-314.

[14] Srivastava H. M. and Singhal J. P., A class of polynomials defined by generalized Rodrigues formula, Ann. Math. Pure Appl., 90(1971), 75-85.

[15] Srivastava R., The inversion of an integral equation involving a general class of polynomials, J. Math. Anal. Appl., 186(1994), 11-20. 